\title{
Photo-induced magnetization enhancement in two-dimensional weakly anisotropic Heisenberg magnets
}

\author{
Antonio Caretta.* Michiel C. Donker, Alexey O. Polyakov, Thomas T. M. Palstra, and Paul H. M. van Loosdrecht \\ Zernike Institute for Advanced Materials, University of Groningen, \\ Nijenborgh 4, 9747 AG Groningen, The Netherlands
}

\begin{abstract}
By comparing the photo-induced magnetization dynamics in simple layered systems we show how light-induced modifications of the magnetic anisotropy directly enhance the magnetization. It is observed that the spin precession in $\left(\mathrm{CH}_{3} \mathrm{NH}_{3}\right)_{2} \mathrm{CuCl}_{4}$, initiated by a light pulse, increases in amplitude at the critical temperature $T_{C}$. The phenomenon is related to the dependence of the critical temperature on the axial magnetic anisotropy. The present results underline the possibility and the importance of the optical modifications of the anisotropy, opening new paths toward the control of the magnetization state for ultrafast memories.
\end{abstract}

Fast and efficient control of the magnetic state of a material requires the understanding of non-equilibrium processes happening in the picosecond time regime. The importance of studying the magnetic phenomena with femtosecond time-resolution was first demonstrated by Beaurepaire et al [1]. In this work it was found that ferromagnetic Ni could be demagnetized with ultrashort optical pulses within one picosecond. Shortly after this observation, a number of experiments showing optical manipulation of the magnetization were published [2 4]. The photo-generation of coherent spin precession as well as the possibility of controlling the spin precession direction via the inverse Faraday effect [5] were demonstrated. These investigations lead finally to the spectacular observation of all-optical magnetization switching in GdFeCo [6]. Recently the field of ultrafast magnetization, "femtomagnetism" [7, started to address increasing attention towards other possible ultrafast switches: propagating acoustic phonons [8, 9] and spin waves [10], or spin polarized electron transport [11] and spin transfer torque [12. All these phenomena are essentially non-equilibrium processes, and the ability to control the magnetic state of a material by optical pulses has obvious potential in applications. The generation of coherent spin precession is a possible method to control the magnetic state of a system.

A spin precession can be initiated by an optical pulse via different mechanisms. Ultrafast demagnetization in metallic thin films can for instance induce a fast $(<10 \mathrm{ps})$ spin reorientation [3] 13. Also the use of circularly polarized optical pulses can efficiently initiate a precession [5, 14]. The first method relies on fast demagnetization rates, and the second on the transparency of the material and strong spin-orbit coupling. In order to investigate the other mechanisms of spin reorientation, we measured two insulating magnetic materials, characterized by a unique range of parameters, low demagnetization rate and nearly quenched spin-orbit coupling. The organic-inorganic hybrid materials realize these requirements, being additionally versatile, tunable and multifunctional [15-17. In particular it would be interesting to investigate the effect of non-equilibrium modifications of the magnetic parameters like the single-ion magnetic anisotropy. The modifications of the magnetic anisotropy show up, phenomenologically, at time scales only limited by the magnetic response of the system, the spin precession period. These processes might be extremely relevant for the understanding all-optical magnetization control, but few experiments address this issue.

In this Letter we report on optical time-resolved Faraday rotation (FR) experiments on simple magnetic systems, the layered organic-inorganic hybrids (OIHs). The magnetic field and temperature dependence of the lightinduced precession is studied in two model systems: ferromagnetic $\left(\mathrm{CH}_{3} \mathrm{NH}_{3}\right)_{2} \mathrm{CuCl}_{4}(\mathrm{MACuCl})$, and antiferromagnetic $\left(\mathrm{CH}_{3} \mathrm{CH}_{2} \mathrm{NH}_{3}\right)_{2} \mathrm{CuCl}_{4}(\mathrm{EACuCl})$. The observed difference in response of the two materials strongly suggests that, even in the low-fluence regime, the magnetization enhancement of the spin precession observed in $\mathrm{MACuCl}$ is due to a non-equilibrium increase of the axial magnetic anisotropy. Before presenting the main results we will first review some basic magnetic properties of the organic-inorganic hybrids.

The layered $\mathrm{Cu}$-based organic-inorganic hybrids here studied are nearly ideal 2-dimensional Heisenberg ferromagnets and have been widely studied in the 70's [18. The structure is constituted by corner sharing $\mathrm{CuCl}_{6}$ octahedra, forming the inorganic layers, and bilayers of organic cations, attached to the octahedra by the $\mathrm{NH}_{3}$ heads [17, 19] (fig. 1b). The proper Hamiltonian to describe the single layer magnetism is [18]:

$$
\mathcal{H}=-2 J \sum_{i>j} \vec{S}_{i} \cdot \vec{S}_{j}+\sum_{i}\left(K S_{y i}^{2}+D S_{z i}^{2}\right)
$$

where $\vec{S}_{i}$ is the spin of the $i$-th site, $S_{y i}$ and $S_{z i}$ respectively the $y$ and $z$ components of the spin, and $K>0$ and $D>0$ express the magnetic anisotropy. The intralayer exchange $J$ is ferromagnetic due to cooperative Jahn-Teller ordering [20], with $J / k \sim 20 \mathrm{~K}$. The exchange interaction between the layers is expressed with $J^{\prime}$ (fig. 


\begin{tabular}{lccccc}
\hline \hline & $T_{C}(\mathrm{~K})$ & $J / k(\mathrm{~K})$ & $J^{\prime} / J$ & $H_{A}^{\text {Ising }} / H_{E}$ & $H_{A}^{X Y} / H_{E}$ \\
\hline $\mathrm{MACuCl}$ & 8.9 & 19.2 & $+5.5 \times 10^{-5}$ & $1.6 \times 10^{-4}$ & $3 \times 10^{-3}$ \\
$\mathrm{EACuCl}$ & 10.2 & 18.6 & $-8 \times 10^{-4}$ & $1.6 \times 10^{-4}$ & $3 \times 10^{-3}$ \\
\hline \hline
\end{tabular}

TABLE I. Parameters characterizing the magnetic phase of $\mathrm{MACuCl}$ and $\mathrm{EACuCl}\left[18\right.$, 27]: the critical temperature $T_{C}$, the intralayer ferromagnetic exchange $J / k \sim 20 \mathrm{~K}$, the interlayer exchange $J^{\prime}$. The planar and the axial anisotropy effective fields, respectively $H_{A}^{X Y} \equiv 2 D S / g \mu_{B}$ and $H_{A}^{I s i n g} \equiv$ $2 K S / g \mu_{B}$, are normalized by the intralayer effective field $H_{E}=2 z J S / g \mu_{B}$, where $z=4$ is the number of neighbors in the layer.

1b), and the Hamiltonian is modified by an additional term accounting for the other layers (see ref. 21]). The interlayer exchange $J^{\prime}$ is up to five orders of magnitude smaller than $J$, and determines the overall three dimensional magnetic character of the compound: while $\mathrm{MACuCl}$ orders ferromagnetically at $8.9 \mathrm{~K}, \mathrm{EACuCl}$ orders antiferromagnetically at $10.2 \mathrm{~K}[18$. It is well known that 2-dimensional Heisenberg magnets do not order at any finite temperature [22]. The only possible cause of ordering in $\mathrm{Cu}$-based $\mathrm{OIHs}$ is the presence of finite interlayer exchange $J^{\prime}$, breaking the 2-d character, or axial magnetic anisotropy $H_{A}^{I s i n g} \equiv 2 K S / g \mu_{B}$ [23]. The preferential axis breaks the continuous symmetry and the system behaves similarly to an Ising 2-d magnet, thus orders at a finite temperature [24 26]. The largest of the deviations from the ideal situation causes magnetic ordering. In table $\mathrm{I}$ the magnetic parameters describing $\mathrm{MACuCl}$ and $\mathrm{EACuCl}$ are shown, with the use of the intralayer effective field $H_{E}=2 z J S / g \mu_{B}(z$ is the number of neighbors in the layer). Specifically for MACuCl: $H_{A}^{I s i n g} / H_{E}>\left|J^{\prime} / J\right|$. Vice versa for EACuCl: $H_{A}^{I s i n g} / H_{E}<\left|J^{\prime} / J\right|$ (see table I). It is very important to recall the dependence of the critical temperature $T_{C}$ on the magnetic anisotropy, when $H_{A}^{I s i n g} / H_{E}>\left|J^{\prime} / J\right|$ 24-26]:

$$
T_{C}=\frac{4 \pi J}{\ln (J / K)} .
$$

It is clear that modifications of $K$, thus $H_{A}^{I s i n g}$, will only affect $\mathrm{MACuCl}$, as instead in $\mathrm{EACuCl}$ the critical temperature is, in first approximation, only a function of $J^{\prime}$ 21.

The samples are grown from solution in an analogy to $\mathrm{PEACuCl}$ (see ref. [19, 28]). Sample thickness is approximately $150 \mu \mathrm{m}$. Magnetic properties are probed by Faraday rotation, at a fixed frequency of $1.55 \mathrm{eV}$ $(800 \mathrm{~nm})$. Time-resolved experiments are performed using a $1 \mathrm{kHz}$ Ti:Sapphire based amplified laser system (Hurricane, Spectra-Physics). The $2.38 \mathrm{eV}(520 \mathrm{~nm})$ pump is generated using a non-linear optical parametric amplifier (Light Conversion). The $520 \mathrm{~nm}$ pulses are optimally tuned to the $\mathrm{Cu}-\mathrm{Cl}$ charge transfer edge [29, 30. The pump fluence used in the experiments
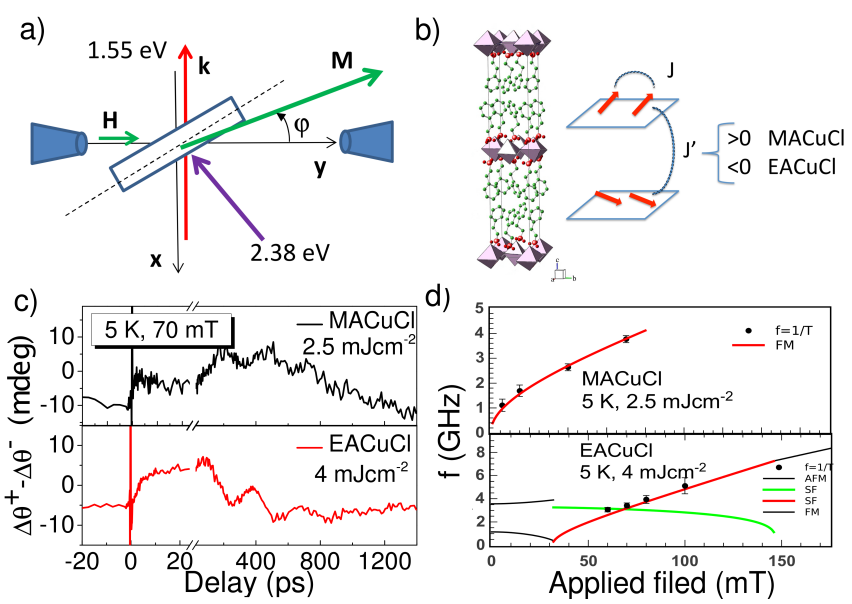

FIG. 1. (Color online) a) Experiment scheme. b) Crystal structure of $\mathrm{EACuCl}$ and exchange constants summary. c) Magnetization dynamics in $\mathrm{MACuCl}$ and $\mathrm{EACuCl}$ at $70 \mathrm{mT}$ and 5 K. d) Precession frequency measured from the Faraday rotation in $\mathrm{MACuCl}$ (up) and $\mathrm{EACuCl}$ (down). The $f(H)$ curves represent the resonance frequency in the antiferromagnetic (AFM), the spin-flip (SF) and the ferromagnetic (FM) phases (EACuCl: more details can be found in Ref. [32]).

ranges from $2.5 \mathrm{mJcm}^{-2}$ to $4 \mathrm{mJcm}^{-2}$, corresponding to a temperature increase of approximately $1 \mathrm{~K}$ (Supplementary data). The time resolution of the experiment is approximately $150 \mathrm{fs}$ 31. Magnetic measurements are performed in tilted-Voigt geometry, with applied magnetic field along $\hat{y}$ perpendicular to the light propagation along $\hat{x}$ (fig. 1a). The linearly polarized pump electric field is oriented along $\hat{z}$, parallel to the crystal optical axis. The samples are rotated of $\varphi_{0} \sim 45^{\circ}$ around the $\hat{z}$ axis. In this configuration the FR is expressed by

$$
\theta \propto \vec{k} \cdot \vec{M} \propto|M| \sin (\varphi)
$$

where $\varphi$ is the angle between the magnetization $\vec{M}$ and $\hat{y}$. The experimental data are represented as $\left(\Delta \theta^{+}-\right.$ $\Delta \theta^{-}$), where $\Delta \theta^{+}$is the FR signal measured at positive applied field $+H$ and $\Delta \theta^{-}$at $-H$. It is easy to note that (Supplementary data)

$$
\Delta \theta^{+}-\Delta \theta^{-} \propto\left(\frac{\Delta M}{M_{0}}+m(T) \Delta \varphi\right) .
$$

where $M_{0}$ is the saturated magnetization measured at $0 \mathrm{~K}, \Delta M$ and $\Delta \varphi$ are the pump induced changes of magnetization and orientation, $m(T) \equiv M(T) / M_{0}$ is the normalized magnetization. It is important to note that at low temperatures, where $m(T) \sim 1$, we are sensitive to changes of $\varphi$ while close to $T_{C}$, where $m(T) \sim 0$, modification of the magnetization absolute value dominates. Because of this at high temperatures variations of magnetization orientation should be enormous to compensate for the low value of $m(T)$.

The magnetization dynamics, in both $\mathrm{MACuCl}$ and $\mathrm{EACuCl}$, is characterized by two time regimes. The time 
dependence of the Faraday rotation at $70 \mathrm{mT}$ and $5 \mathrm{~K}$, in respectively $\mathrm{MACuCl}$ and $\mathrm{EACuCl}$, is shown in figure 1 . At early time-scales, up to 20 ps after the pump arrival, the magnetization increases by approximately $0.5-1 \%$ with a rise time $\tau<10$ ps (Supplementary data). This increase does not depend on the applied field or on the pump polarization. Later times are instead characterized by oscillations, having an amplitude of the same order as the initial increase and a period strongly dependent on the applied magnetic field. The oscillation frequency $f=1 / T$ of $\mathrm{MACuCl}$ and $\mathrm{EACuCl}$ (top and bottom of fig. $1 \mathrm{~d}$ ) at $5 \mathrm{~K}$ is shown as a function of the applied field. The frequencies are obtained from the maxima of the oscillations, by averaging the periods.

The magnetic field dependence of the oscillations matches the precession frequency measured in equilibrium conditions. The increase of the precession frequency $f$ as a function of the applied field $H$ in $\mathrm{MACuCl}$ can be described by $f=\gamma \frac{g}{4 \pi} \sqrt{H\left(H+H_{A}\right)}[3]$, where $\gamma$ is the gyromagnetic factor, $g$ the Landau splitting factor and $H_{A}$ the equilibrium magnetic anisotropy. For $\mathrm{MACuCl}$ an estimate for $H_{A} \sim 200 \mathrm{mT}$ is found, consistent with the static anisotropy field $H_{A}^{X Y}$ reported in literature (see also Table I). For EACuCl the precession frequency matches the antiferromagnetic resonance frequency reported in literature [32], and shown in figure 11. It should be noted that, in both $\mathrm{MACuCl}$ and $\mathrm{EACuCl}$, the amplitude of the precession is comparable to the initial increase of the magnetization and weakly depends on the applied magnetic field.

While the low temperature magnetic field behavior of $\mathrm{MACuCl}$ and $\mathrm{EACuCl}$ is analogous, the response of the two systems is radically different when crossing the critical temperature. The temperature dependence of the magnetization dynamics in $\mathrm{MACuCl}$ (top) and $\mathrm{EACuCl}$ (bottom) is shown in figure 2. The measurements are performed at fixed applied field. It can be easily observed that, while in $\mathrm{EACuCl}$ the magnetic signal simply disappears with increasing temperature, in $\mathrm{MACuCl}$ the precession amplitude grows to a maximum at $9.6 \mathrm{~K}$, close to $T_{C}$. In both compounds the initial increase of the magnetization, between 10 ps and 100 ps, smoothly decreases with increasing temperature. Also the precession frequency, weakly decreasing at higher temperatures, seem to be comparable in the two materials. In order to quantify these observations, we estimate the initial reorientation amplitude by plotting the increase of $\Delta \theta^{+}-\Delta \theta^{-}$at $50 \mathrm{ps}$; the amplitude and the frequency of the precession are estimated from the oscillation maxima. Note that the precession frequencies are normalized by the external magnetic field $H$, as $f / H$. The resulting graphs are shown in figure 3, where $\mathrm{MACuCl}$ and $\mathrm{EACuCl}$ are compared. As expected the behavior of the two compounds is analogous when looking at the normalized precession frequency $f / H$ and the initial reorientation (fig. 3 a and

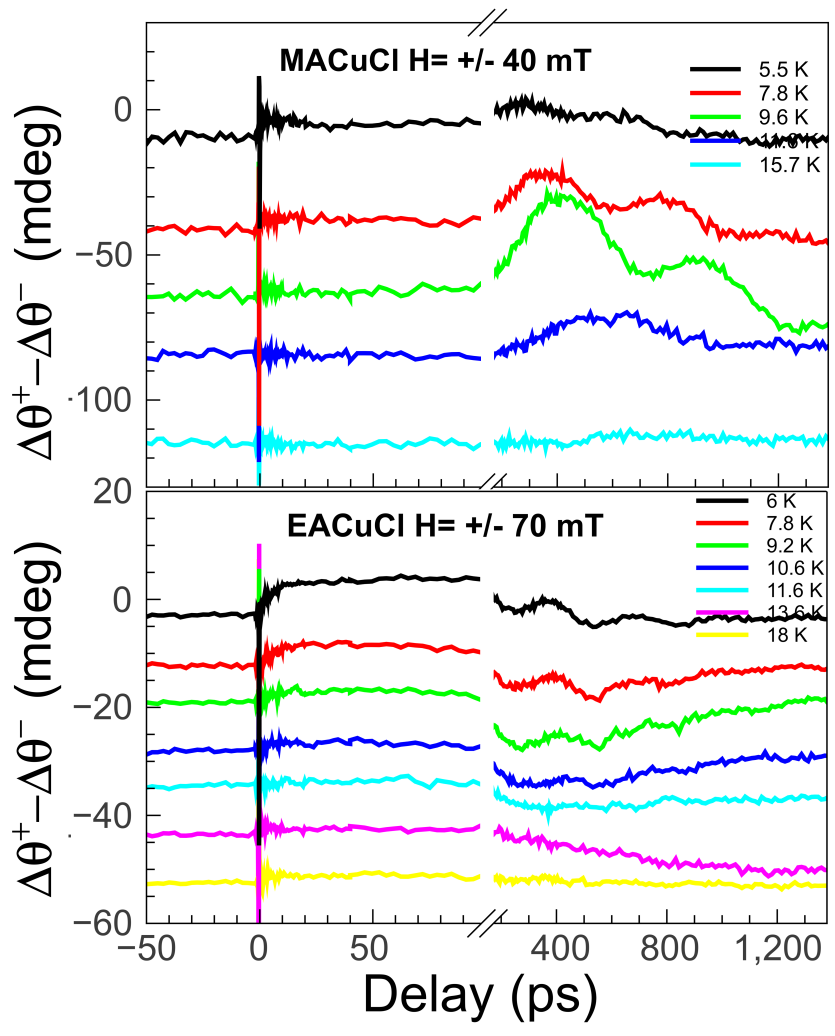

FIG. 2. (Color online) Temperature dependence of the magnetization dynamics in $\mathrm{MACuCl}$ (top, $H= \pm 40 \mathrm{mT}$ ) and $\mathrm{EACuCl}$ (bottom, $H= \pm 70 \mathrm{mT}$ ).

3b). The initial reorientation scales as the spontaneous magnetization $m(T)$, thus sharply decreases as $T_{C}$ is approached from below. It should be noted that this is an indication that the initial dynamics is dominated by the reorientation of the magnetization, rather than a change of its absolute value. In fact, as given in eq. 4, the angular term $\Delta \varphi$ is scaled by $m(T)$ (red curve in fig. $3 \mathrm{p}$ SI 33]). The precession frequency shows a gentle redshift with increasing temperature, in line with temperature dependence of the spin resonance. The most striking difference between $\mathrm{MACuCl}$ and $\mathrm{EACuCl}$ is the amplitude of the oscillation (fig. 3r), which decreases to zero at $T_{C}$ in the latter and grows of a factor of 3, when compared to the value at $4 \mathrm{~K}$, in the former.

The enhancement of the magnetization absolute value observed in $\mathrm{MACuCl}$, and peaked at $T_{C}$, can be explained by a non-equilibrium increase of the axial anisotropy. We assume that the pump pulses produce the same electronic and structural modifications in both compounds at short (50 ps) timescales. This assumption is reasonable since both materials are, in first approximation, a collection of loosely coupled ferromagnetic layers. If one additionally notes that the layers are essentially the same (see table I), the response to the optical excitation, at least within the layers, must be analogous in the two systems. As shown in equation 4 the FR signal is the sum of an 
angular term, proportional to the magnetization $m(T)$, and a term proportional the relative changes of the absolute magnetization $M$. Close to $T_{C} m(T) \Delta \varphi \sim 0$, and the observed changes of FR are dominated by changes of the absolute magnetization $M$. Clearly, although the two systems initially behave similarly (fig. $3 \mathrm{~b}$ ), the absolute magnetization of $\mathrm{MACuCl}$ increases in proximity of $T_{C}$, as shown by the precession amplitude (fig. 3c). The Ising anisotropy is the only layer parameter which, in first approximation, can specifically affect the magnetization dynamics in $\mathrm{MACuCl}$, leaving $\mathrm{EACuCl}$ unperturbed. In fact, assuming sizeable changes of $H_{A}^{I s i n g}$ (thus changes of $K$ in equation 2), only the critical temperature of $\mathrm{MACuCl}$ would have notable modifications. Changes of the critical temperature value are enhanced at $T_{C}$, where larger is the slope of the spontaneous magnetization. This is indeed observed in $\mathrm{MACuCl}$, and it is easy to quantify the effect.

The increase of magnetization in $\mathrm{MACuCl}$, due to an increase of the anisotropy $H_{A}^{I s i n g}$, can be easily estimated from equation 2. The increase of $T_{C}$ in $\mathrm{MACuCl}$ caused by a increase of the anisotropy $K+\Delta K$ is:

$$
T_{C}(K+\Delta K)=T_{C}\left(1+\frac{1}{\ln (J / K)} \frac{\Delta K}{K}\right) .
$$

In the specific case of $\mathrm{MACuCl}$ we have:

$$
\Delta T_{C} \sim \frac{T_{C}}{10} \frac{\Delta K}{K} \sim \frac{\Delta K}{K} .
$$

Additionally the magnetization, because of an increase of $T_{C}$, is enhanced and the effect is larger in particular at the critical temperature, since:

$$
\frac{\Delta M}{M_{0}}=\left|\frac{\partial m}{\partial T}\right| \Delta T_{C} \sim\left|\frac{\partial m}{\partial T}\right| \frac{\Delta K}{K} .
$$

In fig. 3. $\Delta M \propto \frac{\partial m}{\partial T}$ is calculated from the magnetization curve $M$ in applied field (supplementary info) and respectively shown in black and red curves. Given that, close to $T_{C}, \frac{\Delta M}{M_{0}} \sim 0.03$ in $\mathrm{MACuCl}$ and that the maximum of $\frac{\partial m}{\partial T}$ is approximately 0.4 , we obtain an increase of the anisotropy of $\frac{\Delta K}{K} \sim 10 \%$.

Such a small increase of the magnetic anisotropy, which perfectly describes the observations, does not influence the values of the precession frequencies measured at $5 \mathrm{~K}$. In particular it has no role for the magnetization dynamics in $\mathrm{EACuCl}$, and the precession amplitude simply scales as the magnetization $m(T)$, reducing to zero at $T_{C}$ as indeed observed.

We now briefly discuss the possible microscopic origin of the anisotropy enhancement. It should be noted that, because $H_{A}^{I \operatorname{sing}}$ is so small in the hybrids, it is rather difficult to unambiguously determine the origin of the enhancement. Given this, in accordance with [9, the simplest explanation is that the photo generated acoustic
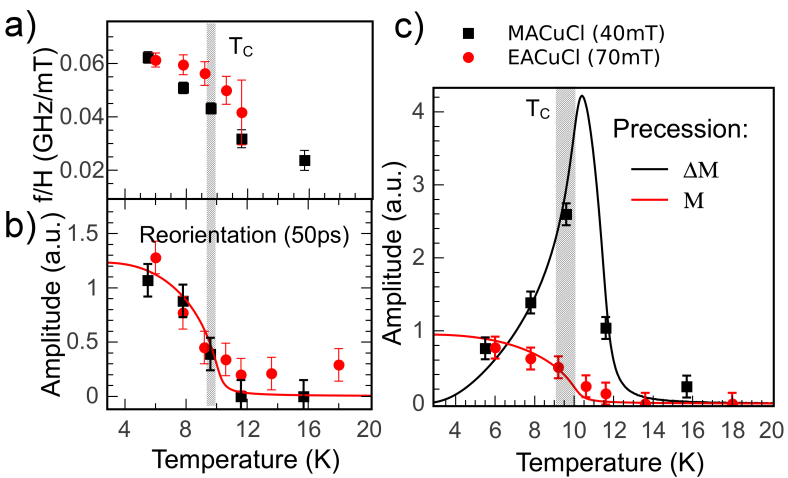

FIG. 3. (Color online) a) Precession frequency (normalized by the applied field); b) reorientation amplitude (at $50 \mathrm{ps}$ delay) and c) precession amplitude in $\mathrm{MACuCl}$ and $\mathrm{EACuCl}$ as a function of temperature. Note that the amplitudes are expressed as $\left(\Delta \theta^{+}-\Delta \theta^{-}\right) /\left(\theta_{0}^{+}-\theta_{0}^{-}\right) . \quad M$ and $\Delta M$ are respectively the magnetization and its derivative calculated at an applied field comparable to the experiment.

phonons distorts the lattice and, due to magneto-elastic coupling, the axial anisotropy is increased. Acoustic phonons are nearly instantaneously created by the pump pulses, and generate a long lasting (400 ps) anisotropic distortion, as indeed observed in our FR experiments.

The effect of anisotropy induced magnetization enhancement can be increased by using multilayer structures based on thin permalloy layers and dielectric spacers. Permalloy materials are well known for the high critical temperature and the high magnetic permeability. These properties result from respectively a large magnetic exchange $J$ and a low single ion anisotropy $K$. As a consequence permalloy materials are good approximations of Heisenberg systems, with critical temperatures up to $T_{C}=k_{B} J \sim 1000 \mathrm{~K}$. As was outlined before, when thin films are formed, the critical temperature is expected to decrease, because of the increasing 2-dimensional character of the system 34. For a thin enough system the equation 2 is applicable and the layer ordering temperature is largely reduced. By using, as interlayer spacer, a material that can induce stress (e.g. piezoelectric) when an external stimulus is applied, the magnetization can be largely enhanced. For instance one monolayer of $\mathrm{Ni}_{83} \mathrm{Fe}_{17}$ orders at approximately $k_{B} J / 3 \sim 850 \mathrm{~K} / 3 \sim 283 \mathrm{~K}[35$. Assuming a relative increase of the anisotropy of $10 \%$ $(\Delta K / K=0.1)$ and $J / K \sim 10^{5}$ as in the hybrids, it is predicted from equation 5 an increase of the critical temperature of $8.5 \mathrm{~K}$. This device would work perfectly in the room temperature regime, where the critical temperature can be optimally tuned by the layer thickness. Additionally the spacer can be selected as a function of different external stimuli, the only condition being a sufficient modification of the anisotropy $K$.

In conclusion the magnetization dynamics in both $\mathrm{MACuCl}$ and $\mathrm{EACuCl}$ can be fully described by a photo- 
induced enhancement of the axial anisotropy, which causes an increase of the magnetization precession amplitude in $\mathrm{MACuCl}$ close to $T_{C}$. The mechanism described in this paper is applicable also to multilayer structures consisting of a combination of piezoelectric layers and magnetic layers with a strain dependent anisotropy, in this way we expect that on can even design devices functional at room temperature.

We acknowledge Prof.Dr. A.V. Kimel, D. Bossini and Dr. R.I. Tobey for useful discussion.

antonio.caretta@elettra.eu

pvl@ph2.uni-koeln.de

[1] E. Beaurepaire, J.-C. Merle, A. Daunois, and J.-Y. Bigot, Phys. Rev. Lett. 76, 4250 (1996).

[2] G. Ju, A. V. Nurmikko, R. F. C. Farrow, R. F. Marks, M. J. Carey, and B. A. Gurney, Phys. Rev. Lett. 82, 3705 (1999)

[3] M. van Kampen, C. Jozsa, J. T. Kohlhepp, P. LeClair, L. Lagae, W. J. M. de Jonge, and B. Koopmans, Phys. Rev. Lett. 88, 227201 (2002).

[4] A. V. Kimel, A. Kirilyuk, A. Tsvetkov, R. V. Pisarev, and T. Rasing, Nature 429, 850 (2004).

[5] A. V. Kimel, A. Kirilyuk, P. A. Usachev, R. V. Pisarev, A. M. Balbashov, and T. Rasing, Nature 435, 655 (2005)

[6] C. D. Stanciu, F. Hansteen, A. V. Kimel, A. Kirilyuk, A. Tsukamoto, A. Itoh, and T. Rasing, Phys. Rev. Lett. 99, 047601 (2007).

[7] A. Kirilyuk, A. V. Kimel, and T. Rasing, Rev. Mod. Phys. 82, 2731 (2010)

[8] J.-W. Kim, M. Vomir, and J.-Y. Bigot, Phys. Rev. Lett. 109, 166601 (2012)

[9] O. Kovalenko, T. Pezeril, and V. V. Temnov, Phys. Rev. Lett. 110, 266602 (2013).

[10] T. Satoh, Y. Terui, R. Moriya, B. A. Ivanov, K. Ando, E. Saitoh, T. Shimura, and K. Kuroda, Nat Photon 6, 662 (2012).

[11] A. Eschenlohr, M. Battiato, P. Maldonado, N. Pontius, T. Kachel, K. Holldack, R. Mitzner, A. Fölisch, P. M. Oppeneer, and C. Stamm, Nat Mater 12, 332 (2013).

[12] P. Nemec, E. Rozkotova, N. Tesarova, F. Trojanek, E. De Ranieri, K. Olejnik, J. Zemen, V. Novak, M. Cukr, P. Maly, and T. Jungwirth, Nat Phys 8, 411 (2012).

[13] The change of the shape anisotropy is the nonequilibrium condition for precession: the reorientation of the equilibrium axis is as fast as the demagnetization in the material.

[14] D. Bossini, A. M. Kalashnikova, R. V. Pisarev, T. Rasing, and A. V. Kimel, Phys. Rev. B 89, 060405 (2014).

[15] D. B. Mitzi, Prog. Inorg.Chem. 48, 1 (1999).

[16] C. N. R. Rao, A. K. Cheetham, and A. Thirumurugan, J. Phys.: Condens. Matter 20, 083202 (2008).

[17] A. Caretta, R. Miranti, R. W. A. Havenith, E. Rampi, M. C. Donker, G. R. Blake, M. Montagnese, A. O. Polyakov, R. Broer, T. T. M. Palstra, and P. H. M. van Loosdrecht, Phys. Rev. B 89, 024301 (2014)

[18] L. J. de Jongh and A. R. Miedema, Adv. Phys. 23, 1 (1974).

[19] A. O. Polyakov, A. H. Arkenbout, J. Baas, G. R. Blake, A. Meetsma, A. Caretta, P. H. M. van Loosdrecht, and T. T. M. Palstra, Chemistry of Materials 24, 133 (2012)

[20] D. Khomskii and K. Kugel, Solid State Commun. 13, 763 (1973)

[21] L. J. de Jongh and H. E. Stanley, Phys. Rev. Lett. 36, 817 (1976)

[22] N. D. Mermin and H. Wagner, Phys. Rev. Lett. 17 (1966).

[23] For $D \gg K$ the layer has planar anisotropy, and for $D \simeq K$ axial anisotropy. The magnetic system can be described respectively with a XY model or a Ising model, thus the names $H_{A}^{X Y}$ and $H_{A}^{I s i n g}$.

[24] M. Bander and D. L. Mills, Phys. Rev. B 38, 12015 (1988).

[25] R. P. Erickson and D. L. Mills, Phys. Rev. B 43, 11527 (1991)

[26] E. Kuz'min and G. Petrakovskii, Soviet Physics Journal 31, 256 (1988).

[27] A. H. Arkenbout, Organic-Inorganic Hybrids, Ph.D. thesis, University of Groningen, the Netherlands (2010).

[28] H. Arend and W. Huber, J. Cryst. Growth 43, 213 (1978).

[29] G. Heygster and W. Kleemann, Physica B\&C 89, 165 (1977).

[30] A. Caretta, R. Miranti, A. H. Arkenbout, A. O. Polyakov, A. Meetsma, R. Hidayat, M. O. Tjia, T. T. M. Palstra, and P. H. M. v. Loosdrecht, Journal of Physics: Condensed Matter 25, 505901 (2013)

[31] The cross-correlation had been measured on a ZnSe nonlinear crystal and on the sample itself.

[32] M. Tanimoto, T. Kato, and Y. Yokozawa, Physics Letters A 58, 66 (1976)

[33] $m(T)$ is the magnetization calculated in presence of a small magnetic field, as in the experiments.

[34] F. Huang, M. T. Kief, G. J. Mankey, and R. F. Willis, Phys. Rev. B 49, 3962 (1994)

[35] J. Dubowik, F. Stobiecki, and T. Luciński, Phys. Rev. B 57, 5955 (1998). 